\title{
Role Conversion Considering Its Context and Syntactic Property
}

\author{
Young-Shing Youn ${ }^{1,2}$, Hye-Jeong Song ${ }^{1,2}$, Chan-Young Park ${ }^{1,2}$, Jong-Dae Kim ${ }^{1,2}$ \\ and Yu-Seop Kim ${ }^{1,2 *}$ \\ ${ }^{1}$ Dept. of Convergence Software, Hallym University, Korea \\ ${ }^{2}$ Bio-IT Research Center, Hallym University, Korea \\ Pour657@naver.com, \{hjsong, cypark,kimjd,yskim01\}@hallym.ac.kr
}

\begin{abstract}
Semantic Role Labeling (SRL) is to determine the relationship between predicates and their arguments in a sentence. In order to determine the semantic roles, a large amount of corpus with annotated semantic roles is required. Nowadays the most widely used semantic corpus is Proposition Bank (PropBank) which is semantically annotated over the predicate and argument structure. But the Korean version of the PropBank could not be widely used because the corpus has limitation in size and be different from its original English version in its usability. To solve these problems, we also used another semantic tagged corpus, built by Sejong Plan, which is nation-wide Korean corpus construction project. However, the task of corpus construction with semantic roles defined in PropBank and Sejong is much time-consuming and these corpora use their own role sets. They finally require a way of converting one role to other side role(s). In this paper, we propose a method for automatically converting the roles. First, we use similarity between a given noun argument word to find a new role and noun words appearing in the example sentences of candidate roles. Second, we extract suffix of the argument word and estimate closeness between the suffix and candidate roles. Finally, the predicate itself is used for selection, that is we calculate the closeness between the predicate and the candidate roles. With these, the role is decided among multiple candidate roles. In the experiment, we convert 491 arguments automatically and about $78 \%$ of them show the agreement with manually annotated arguments.
\end{abstract}

Keywords: Semantic role, Automatic conversion, Semantic role conversion, Similarity estimation, Probability table

\section{Introduction}

Semantic Role Labeling is a process that determines the relationship between the meanings of predicate-arguments in sentences [1]. A large number of corpora that are tagged with semantic roles are necessary for Semantic Role Labeling [2]. In Korean, many studies that uilize the corpus that defined as semantic role at Sejong Plan [3][4]. But the world-wide most commonly used corpus is the Proposition Bank (hereafter termed "PropBank") [5].

PropBank refers to a corpus in which the predicate-argument structure is tagged. This corpus was mainly constructed in English and Chinese. However, the Korean PropBank has much fewer learning materials than the corpus of English and Chinese so that its accuracy is relatively low, and the use of the Korean PropBank for semantic role labeling may cause many problems as the special characteristics of the Korean language are not reflected. To solve this problem, we used the tagged Sejong corpus (to be termed "Sejong"), defined in the Sejong Plan, which specializes in Korean [6]. Construction of the Sejong sense-tagged corpus is important as a research related to the semantic role

${ }^{*}$ Corresponding Author 
decisions of Korean have, in the main, used the Sejong sense system. However, significant time and cost have been spent on constructing the PropBank semantic role and Sejong semantic role tagged corpora as the work is conducted manually. Considering this, a new method is necessary to automatically convert the new sense-tagged corpus to the existing sense-tagged corpus.

To achieve this, a method to automatically convert the argument tagged with Sejong semantic role to the Korean PropBank semantic role is presented in [7]. Methods of measuring the mean degree of similarity between the concept of the trunk-based on the measurement method [8][9], information content (IC) measurement method [10][11], the defective hybrid measurement of two methods [12-15] could be considered as a tool for converting roles. For the automatic conversion, Argument sense similarity estimation, which is the subject of the semantic role change, is calculated in [7]. Conversion is made against specific semantic roles from the existing data by identifying the semantic role of words whose sense is similar to the converting argument. In this case, only the argument itself is considered, and its context information is excluded. A problem existed as the hierarchical structure information was not reflected in the automatic conversion method for the proposed semantic role.

In this study, the similarity between argument words was calculated by giving a weight for each level against the length of the path of the noun class that is formed, like each tree, through reflection of the hierarchical structure of the two nouns. Based on the results of the calculation, the automatic conversion method that considered both a Josa and the predicate, which are assumed to have had a close relationship with the argument word, is presented.

In chapter 2, the interrelationship between the Propbank and Sejong semantic role is investigated, and a method to automatically convert the Propbank to Sejong semantic role is provided in chapter 3 . In chapter 4 , the result of the comparison between corpus tagged with the use of the semantic role conversion method proposed here and corpus tagged by the existing users are explained. Finally, chapter 5 contains the conclusion and future research.

\section{Interrelationship between the PropBank and Sejong Semantic Roles}

The sense tagged corpus constructed in [16] was tagged with the PropBank semantic roles and the Sejong semantic roles. [Table 1] shows the mapping satatus of the PropBank semantic roles, which are extracted from 10,263 Korean PropBank sentences, to the Sejong semantic roles. Each line of [Table 1] shows the PropBank semantic role, Sejong semantic role, and the percentage count and weight of the candidate Sejong semantic role that is mapped onto the PropBank semantic role.

Table 1. PropBank Semantic Role and Sejong Candidate Semantic Role

\begin{tabular}{|c|c|c|}
\hline PropBank & Sejong & Count(\%) \\
\hline ARG0 & AGT & $6172(76 \%)$ \\
\hline \multirow{2}{*}{ ARG1 } & THM & $19912(90 \%)$ \\
\hline \multirow{2}{*}{ ARG2 } & FNS & $1119(26 \%)$ \\
\cline { 2 - 3 } & GOL & $1067(24 \%)$ \\
\cline { 2 - 3 } & EFF & $826(19 \%)$ \\
\hline
\end{tabular}




\begin{tabular}{|c|c|c|}
\hline ARG3 & GOL & $544(60 \%)$ \\
\hline \multirow{2}{*}{ M-ADV } & EFF & $551(69 \%)$ \\
\hline & CNT & $161(20 \%)$ \\
\hline M-CAU & EFF & $487(81 \%)$ \\
\hline \multirow{2}{*}{ M-CND } & EFF & $36(32 \%)$ \\
\hline & CNT & $30(27 \%)$ \\
\hline M-DIR & DIR & $195(80 \%)$ \\
\hline \multirow{2}{*}{ M-DIS } & EFF & $120(43 \%)$ \\
\hline & CNT & $82(29 \%)$ \\
\hline \multirow{2}{*}{ M-EXT } & CRT & $330(52 \%)$ \\
\hline & EFF & $199(31 \%)$ \\
\hline M-INS & INS & $794(97 \%)$ \\
\hline M-LOC & LOC & $3274(86 \%)$ \\
\hline M-MNR & INS & $1470(75 \%)$ \\
\hline \multirow{2}{*}{ M-PRD } & INS & $159(50 \%)$ \\
\hline & EFF & $95(30 \%)$ \\
\hline M-PRP & PUR & $146(91 \%)$ \\
\hline M-TMP & LOC & $1107(78 \%)$ \\
\hline
\end{tabular}

The relationship between the PropBank and Sejong semantic roles is reanalyzed as more detailed analysis was necessary using more data than the 3,759 that were previously used for the final analysis. When the Sejong Candidate semantic role is 1:1, like ARG0, ARG1, and M-CAU, the semantic role is determined immediately, but there is ambiguity when the candidate semantic role is plural as in ARG2, M-ADV, and M-CND. To solve this ambiguity, Similarity Estimation is calculated using the weight of the level of noun class of the argument of each semantic role. By converting this to the value of the combination probability table, the semantic role is finally determined through calculation between the PropBank semantic role that is subject to the conversion and the Sejong Candidate semantic role. 


\section{Automatic Conversion of Semantic Role}

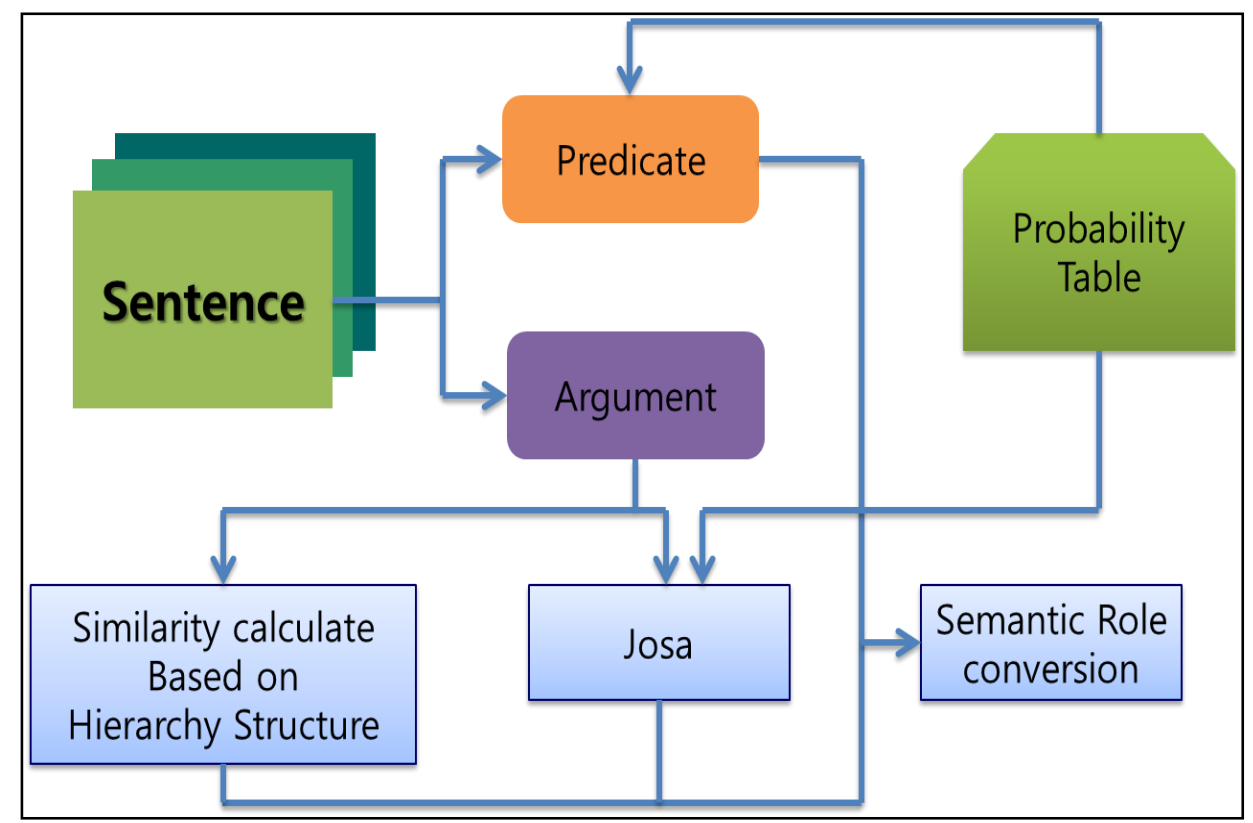

Figure 1. Process of Conversion of Semantic Role

Figure 1 shows the process of automatic conversion of the semantic role from the PropBank to Sejong. Sentences are divided into argument and predicate. The semantic role in [17] is converted by calculating the similarity between the argument that has a semantic role, which is subject to conversion, and the other argument, which has the conversion candidate semantic role. However, the accuracy of this method is low as it only compares the similarity of the argument itself, but does not consider the contents in their context. Nor does it apply noun hierarchical information. When the argument is used as a subject or object, semantic role conversion is simple as the PropBank semantic role and Sejong semantic role have 1:1 mapping; however, there is a different semantic role according to the Josa and predicate in the case of adverbial Josa. As a result, to calculate the similarity between the semantic role that is subject to conversion and the candidate semantic role, a weight should be assigned to each level by reflecting the noun hierarchical structure information for the argument. Furthermore, accurate semantic role conversion can be conducted using the predicate of the argument and the semantic role combination table of Josa.

\subsection{Calculation of Distance between Noun Class with Use of Weight for Each Level}

Figure 2 shows the hierarchical structure of the CBL1, and each number provides information related to the location within the concept system, which is the higher concept and step information. The digits of the concept number show the steps, and the number with a final digit deleted is included in the higher concept. In Figure 2., as a higher concept of 1112, which shows jo-jic 'Organization,' 11 is gu-chea 'Concreteness' as a higher concept than the 111, which indicates ju-chea 'Main agent.' 


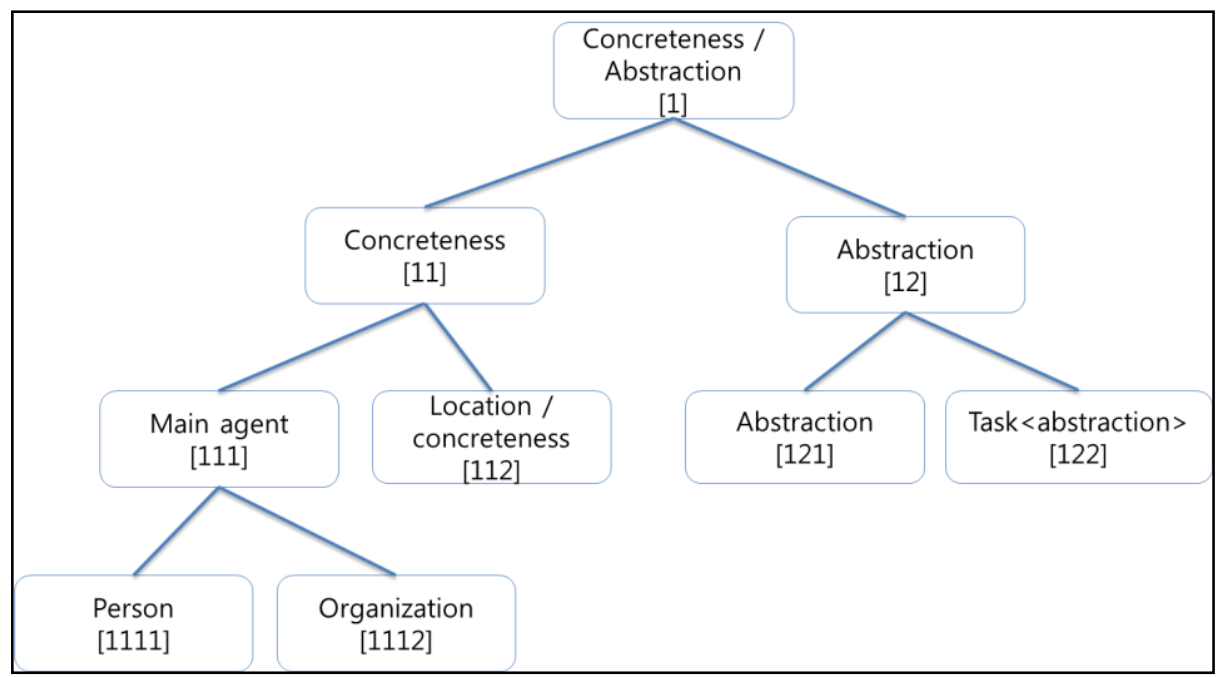

Figure 2. Koeran Noun Lexical Semantic Network(CBL1)

This study calculates the similarity by applying the weight for each level between the noun classes. When the hierarchical structure is not considered, the edge between 1112 and 111 , between 111 and 11 , and between 11 and 112 is calculated to acquire the value 3. As the number of the class reduces, the class becomes higher. By using this, weight is applied to reduce the similarity value for each level of class of the noun that has a tree structure. When the top class differs, the value is calculated by reducing the similarity value by 1 and by $1 / 2$ as it goes to the lower class. By providing weight, the calculation of similarity between classes 112 and 1112 resulted in 0.375 . The formula to calculate the distance between each class is as in the following Figure 3 .

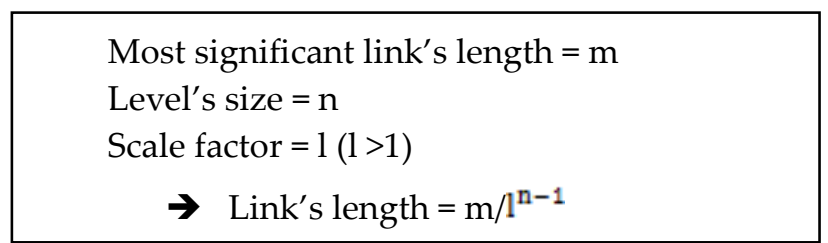

Figure 3. Calculation of the Distance between Classes with Reflection of the Level on the Hierarchical Structure

\subsection{Probability Table Predicate with the Use of Josa Information}

Normal sentences are composed of arguments and predicates. When the argument is used as a subject or object, it is mapped with 1:1 ratio between PropBank ARG0 and ARG1 and between Sejong AGT and THM. In the case of an adverb that has a subject and object, the semantic role is tagged using a completely different method according to the Josa and predicate of the argument.

hea-byeon(beach)[verb-impaired common noun]+ -eh[adverb Josa] LOC(LOC) hea-byeon(beach)[ verb-impaired common noun]+ -ro[adverb Josa] ARG3(GOL) hea-byeon(beach)[ verb-impaired common noun]+ -eseo[adverb Josa] ARG2(SRC)

Figure 4. Semantic Role Corpus according to Josa and Argument 
Figure 4 shows that the PropBank semantic role and Sejong semantic role are converted in a completely different way according to the Josa and predicate of the argument. When the Josa -ro is tagged to the noun hea-byeon 'beach' and predicate, and when -eh is tagged to the ARG3 and GOL, which shows the arrival point, M-LOC and LOC, which indicate location, are mapped. When -eseo is tagged, it is mapped as ARG2 and SRC, which indicate the starting point. As such, the semantic role is determined in a completely different way according to the Josa and predicate, and the accuracy decreases. To accurately define the semantic role, this study provides a combination probability table that contains information about each Josa and predicate.

To prepare data for the table, this study uses a syntax tagged corpus prepared by the Electronics and Telecommunications Research Institute (ETRI) to tag semantic role for the predicate-argument of 12,000 sentences. Following this semantic role tagging, the probability table is constructed with 4,414 tables for verbs and Josa is constructed with 241 tables. Each table stores information about the Sejong semantic roles that are used for the 12,000 data sentences.

Table 2. Probability Relation of Josa -eh and -gachi

\begin{tabular}{|c|c|c|}
\hline \multirow{2}{*}{ Josa } & $\begin{array}{c}\text { Sejong } \\
\text { Semantic } \\
\text { Role }\end{array}$ & Probability \\
\hline \multirow{2}{*}{-eh } & LOC & 0.43 \\
\cline { 2 - 3 } & GOL & 0.26 \\
\hline \multirow{3}{*}{-gachi } & EFF & 0.56 \\
\cline { 2 - 3 } & FNS & 0.06 \\
\cline { 2 - 3 } & COM & 0.17 \\
\hline
\end{tabular}

Table 2 shows part of the probability table that contains information about the Josa -eh and gachi. The Josa -eh is used as LOC, which indicates the location in the Sejong semantic Role, and GOL, which indicates the arrival point. The result shows that Josa -eh is often used for location and for the arrival point according to the arrival point. The Josa gachi 'together' is used as an EFF, which indicates effector, FNS, which indicates the status of the result, and COM, which indicates a companion and shows that this is used as an effector. 


\section{Table 3. Probability Relation of Verbs 'Be Based' and 'See'}

\begin{tabular}{|c|c|c|}
\hline Predicate & $\begin{array}{c}\text { Sejong } \\
\text { Semantic } \\
\text { Role }\end{array}$ & Probability \\
\hline \multirow{3}{*}{ Be based } & THM & 0.33 \\
\cline { 2 - 3 } & LOC & 0.67 \\
\hline \multirow{2}{*}{ See } & AGT & 0.12 \\
\cline { 2 - 3 } & THM & 0.51 \\
\cline { 2 - 3 } & LOC & 0.14 \\
\hline
\end{tabular}

Table 3 shows a higher semantic role in the probability table of the verbs 'be based' and 'see.' 'Be based' uses LOC, which often indicates location for the Sejong semantic role. In the case of 'See,' mapping was conducted for AGT, which indicates agent, THM, which indicates object, and LOC, which indicates location, and it shows that this is used as a meaning of the object. Accordingly, the semantic role can be used differently according to the argument, Josa, and predicate of the probability table, and is necessary for the semantic role conversion of the argument.

\subsection{Method of Automatic Conversion of Heterogeneous Semantic Role Tagging with Reflection of Noun Hierarchical Structure}

Let us assume that the PropBank semantic role, which is subject to conversion, is Prop and the two conversion candidate Sejong semantic roles are S1 and S2. Let us also assume that the Prop and S1 groups are Dset1 and the Prop and S2 groups are Dset. The value that only contains $P$ is termed $p$.

(1) The concept number of each word is determined by applying the words included in the value result from the value that is tagged to Dset1 and Dset2 by applying them to the hierarchical concept system.

(2) To automatically convert the semantic role, which is tagged to P, to S1 or S2, five groups are generated by randomly selecting 150 numbers from the numbers tagged to each Sejong semantic role.

(3) Taking the words included in Group 1 as test data, Similarity Estimation between the words included in Groups 2, 3, 4, and 5 is calculated. In the same way, Similarity Estimation is calculated for other words of other groups.

(4) Let us assume that the two calculated values of Similarity Estimation are R1 and $\mathrm{R} 2$.

(5) Take the value from the predicate that is tagged to Dset 1 and the combination probability table of Josa. In the same way, take the values for Dset 2.

(6) Add the probability value of R1 to Dset1 and do the same for R2 and Dset2.

(7) Convert the semantic role to the group that has the smallest value from the result of summation.

The purpose of randomly selecting 150 sentences is to test using the same numbers as the result, which may deflect to one direction when there is a large difference in the relation analysis from the PropBank semantic role to the Sejong semantic role. 


\section{Test}

This study uses the Syntax Tagged Corpus prepared by the ETRI as the sentence data to investigate the relationship to the predicate-argument. To conduct a test, a sentence is used as an example: 'For this reason, to use the word 'Realism,' it should be used when it is viewed as based on the actual situation according to our views as it is in the classical realism of the 19th century." A test for semantic role conversion is conducted using the method described in Chapter 4.
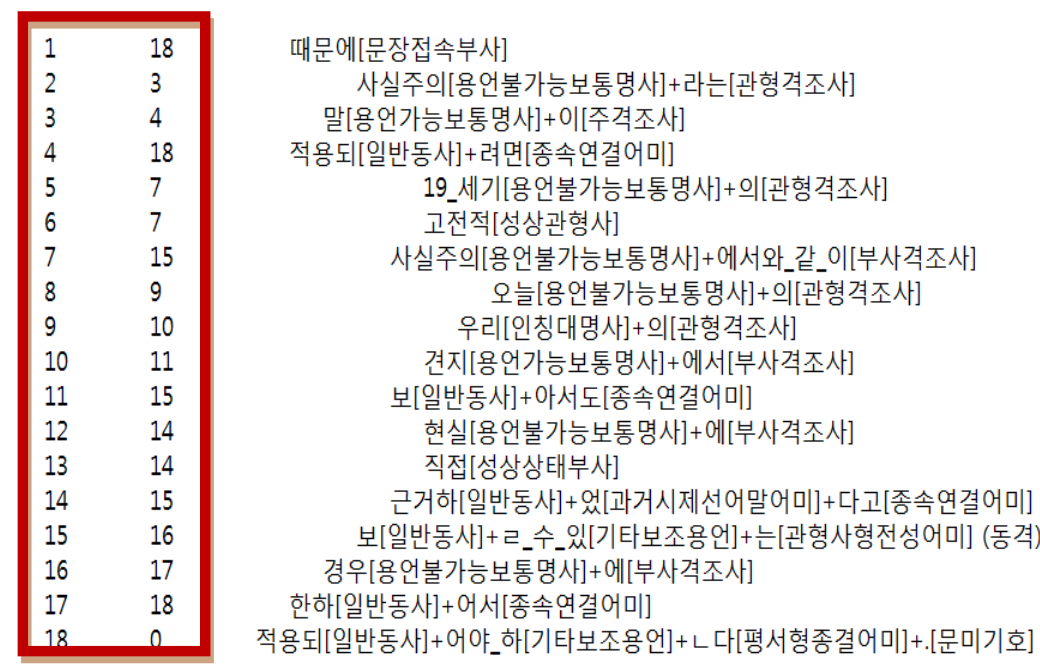

Figure 5. Example of Predicate - Argument Relation

In Figure. 5, the interdependency relation between argument and predicate is expressed with two indexes where the first index for each line is the sequence of word-phrase and the second index is the sequence of interdependent word-phrase. It is expressed that wordphrase is under an interdependent relation when the first and second indices from each line are the same as each other. As an example, the arguments "Because," "If it is to be applied," and "Provided that" have a relation to the predicate "It should be applied." If it is separate to the argument and predicate in consideration of the interdependency between the argument and predicate to convert such a semantic role of the argument, the result comes as in Table 4. The argument is separated into noun + Josa, and each argument is tagged with a PropBank semantic role.

As in Table 4., the PropBank semantic role and Sejong semantic role are mapped at a ratio of $1: 1$ in the mal $+-\mathrm{e}$, and geon-ji + -eso and semantic role is converted to ARG1(THM), LOC(LOC). However, semantic role conversion, with the use of the semantic role similarity estimation and probability table, is necessary as the PropBank semantic role and Sejong semantic role are mapped at a ratio of 1:N in the hyun-sil + -eh, and kyung-wu +- eh. When the values are added through calculation of the similarity between the PropBank semantic role and Sejong semantic role for each argument and by the use of predicate and complex Josa probability tables, the result emerges as in Table 5.

According to Table 5, hyunsil + eh is ARG2(LOC), sasiljueui + ehseowa gachi is $\mathrm{ADV}(\mathrm{EFF})$, and gyeongwoo + eh is ARG2(LOC), taking the smallest value, and the PropBank semantic role is converted to each Sejong semantic role. 
Table 4. Example of Result of Separation of Argument and Predicate

\begin{tabular}{|c|c|c|}
\hline Argument & $\begin{array}{c}\text { Semantic } \\
\text { Role }\end{array}$ & $\begin{array}{c}\text { Sejong Candidate } \\
\text { Semantic Role }\end{array}$ \\
\hline mal +-e & ARG1 & THM \\
\hline geon-ji + -eseo & M-LOC & LOC \\
\hline hyun-sil + -eh & ARG2 & FNS, GOL, LOC \\
\hline $\begin{array}{c}\text { sasilhueui + } \\
\text { ehseowa gachi }\end{array}$ & ADV & EFF, CNT \\
\hline gyeongwoo + -eh & ARG2 & FNS, GOL, LOC \\
\hline
\end{tabular}

Table 5. Semantic Role Calculation Results using Predicate and Josa Probability Table

\begin{tabular}{|c|c|c|c|c|}
\hline Argument & PropBank & Sejong & Predicate & Sum \\
\hline hyun-sil + -eh & ARG2 & GOL & Be based & 0.483 \\
\hline hyun-sil + -eh & ARG2 & FNS & Be based & 0.55 \\
\hline hyun-sil + -eh & ARG2 & LOC & Be based & 0.274 \\
\hline $\begin{array}{c}\text { sasilhueui + } \\
\text { ehseowa gachi }\end{array}$ & ADV & EFF & See & 0.05 \\
\hline $\begin{array}{c}\text { sasilhueui + } \\
\text { ehseowa gachi }\end{array}$ & ADV & CNT & See & 0.16 \\
\hline $\begin{array}{c}\text { gyeongwoo + } \\
\text {-eh }\end{array}$ & ARG2 & GOL & Limit & 0.26 \\
\hline $\begin{array}{c}\text { gyeongwoo + } \\
\text {-eh }\end{array}$ & ARG2 & FNS & Limit & 0.34 \\
\hline $\begin{array}{c}\text { gyeongwoo + } \\
\text {-eh }\end{array}$ & ARG2 & LOC & Limit & 0.15 \\
\hline
\end{tabular}

Table 6. The Result of Similarity between the User-tagged Corpus and the Automatic Tagged Corpus

\begin{tabular}{|c|c|c|c|}
\hline Argument & User-tagged & $\begin{array}{c}\text { Automatic- } \\
\text { tagged }\end{array}$ & Result \\
\hline mal + -e & ARG1(THM) & ARG1(THM) & $\circ$ \\
\hline geon-ji + -eseo & $\begin{array}{c}\text { M- } \\
\text { LOC(LOC) }\end{array}$ & M-LOC(LOC) & $\circ$ \\
\hline hyun-sil + -eh & ARG2(LOC) & ARG2(LOC) & $\circ$ \\
\hline $\begin{array}{c}\text { sasilhueui + } \\
\text { ehseowa gachi }\end{array}$ & M-ADV(EFF) & M-ADV(EFF) & $\circ$ \\
\hline $\begin{array}{c}\text { gyeongwoo + } \\
\text {-eh }\end{array}$ & ARG2(CRT) & ARG2(LOC) & X \\
\hline
\end{tabular}

Table 6 . shows the results of similarity between the user-tagged corpus and the other corpus in which the automatic conversion method is applied. The latter showed four similarities and one difference compared to the manually tagged corpus. 
We showed $78 \%$ of agreement between manual tagging and automatic conversion after experiment with 100 ETRI sentences.

\section{Conclusions}

In this paper, we propose an automatic conversion of semantic roles from PropBank to Sejong corpus to recover the problem caused from original manual annotation work. We also build probability relation tables of Josas and predicates to decide accurate semantic roles. To convert PropBank semantic roles to Sejong semantic roles, firstly we should calculate the similarity between semantic roles to be converted and the candidate semantic roles by using hierarchical structure with weights of its level. Then we decide the final semantic role by using the josa and predicate tables. For the experiment, we compared manually annotated roles and automatically converted roles. About $78 \%$ of roles are correctly converted when 100 test data are used.

For the future, we have a plan to develop a methodology to automatically annotate the PropBank semantic role and Sejong semantic role. And besides nouns, josas, and their predicates, other constituents of the sentence will be utilized for the more accurate semantic role labeling system.

\section{Acknowledgments}

This paper is a revised and expanded version of a paper entitled "Automatic Semantic Role Conversion based on Word Similarity and Statistics" [17] presented at SIT 2016, Jeju, Korea, December 21-23.

This research was supported by Basic Science Research Program through the National Research Foundation of Korea (NRF) funded by the Ministry of Science, ICT and future Planning (2015R1A2A2A01007333), and by Hallym University Research Fund, 2015 (HRF-201512-013).

\section{References}

[1] S. Kang and J. Park, "Determination of Thematic Roles according to Syntactic Relations Using Rules and Statistical Models in Korean Language Processing," Korea Society of industrial information systems., vol. 8, no. 4, (2003), pp. 33-42.

[2] M. Lee, Y. Lee, J. Lee and Y. Kim, "Korean Semantic Tagged Corpus Construction working," Proceedings of the $24^{\text {th }}$ Annual Conference on Human and Cognitive Language Technology, Busan, Korea, October, (2012), pp-99-103.

[3] B. Kim, Y. Lee and J. Lee, "Unsupervised Semantic Role Labeling for Korean Adverbial Case," Journal of The Korean Institute of Information Scientists and Engineers., vol. 34, no. 2, (2007), pp.112-122

[4] B. Kim, Y. Lee, S. Na, J. Kim and J. Lee, "Bootstrapping for Semantic Role Assignment of Korean Case Marker," Proceedings of the 2006 Korea Computer Congress, Yongpyeong, Korea, June, (2006), pp.4-6.

[5] M. Palmer, P. Kingsbury and D. Gildea, "The Proposition Bank: An Annotated Corpus of Semantic Roles," Computational Linguistics., vol. 31, no. 1, (2005), pp.71-106.

[6] J. Choe, "Utilizing the Korean language sources: Sejong Project," Proceedings of the Corpus Methodology Workshop, Seoul, Korea, (2007).

[7] J. Cho, H. Jeong and Y. Kim, "Development of Automatic Predicate-argument Analysis System for Korean Tagged Corpus," Information Processing Society Journal B., vol. 19-B, no. 1, (2012), pp.43-52.

[8] P. Resnik, "Using Information Content to Evaluate Semantic Similarity in a Taxonomy." Proceedings of the 14th IJCAI, Montreal, Canada, August, (1995), pp. 228-453.

[9] J. Jiang and D. Conrath, "Semantic Similarity based on corpus statistics and lexical taxonomy," Proceedings of the International Conference on Research in Computational Linguistics, Taipei, Taiwan, August, (1997), pp. 19-33.

[10] D. Lin, "Using syntactic dependency as a local context to resolve word sense ambiguity," Proceedings of the 35th Annual Meeting of the Association for Computational Linguistics, Madrid, Spain, July, (1997), pp. 64-71.

[11] M. Rodriguez and M. Egenhofer, "Determining semantic similarity among entity classes from different ontologies," IEEE Transactions on Knowledge and Data Engineering., vol. 15, no. 2, (2003), pp. 442456. 
[12] G. M. Euripides and G. Petrakis, "Design and evaluation of semantic similarity measures for concepts stemming from the same or different ontologies," Proceedings of the 4th Workshop on Multimedia Semantics (WMS'06), Chania, Greece, June, (2006), pp. 44-52.

[13] F. Lin and K. Sandkuhl, "A Survey of Exploiting WordNet in Ontology Matching," Proceedings of the IFIP, Warsaw, Poland, September, (2008), pp. 341-350.

[14] V. Cross and X. Hu, "Using Semantic Similarity in Ontology Alignment", Proceedings of the $6^{\text {th }}$ International Conference on Ontology Matching - Volume 814, Bonn, Germany, October, (2011), pp. 61-72.

[15] M. Seok, Y. Youn and Y. Kim, “Automatic Transformation of Heterogeneous Semantic Roles using Similarity Degree in Hierarchical Concept Structure," Proceedings of the Korea Computer Congress, Busan, Korea, June, (2014), pp.1773-1775.

[16] Y. Kim, H. Chae, B. Snyder and Y. Kim, "Training a Korean SRL System with Rich Morphological Features," Proceedings of the 52nd Annual Meeting of the Association for Computational Linguistics, Baltimore, MD., USA., June, (2014), pp.637-642.

[17] Y. Young, H. Song, J. Kim, C. Park and Y. Kim, "Automatic Semantic Role Conversion based on Word Similarity and Statistics," Advanced Science and Technology Letters, vol. 142 (SIT 2016), (2016), pp. 92-95.

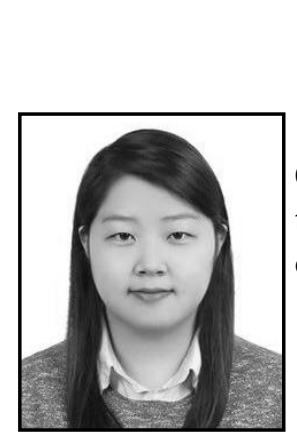

\section{Authors}

Young-Shin Youn, she received the B.S. from the Department of Convergence Software in Hallym University. She currently studies in the same department for her MS course. Her research interests focus on natural language processing.

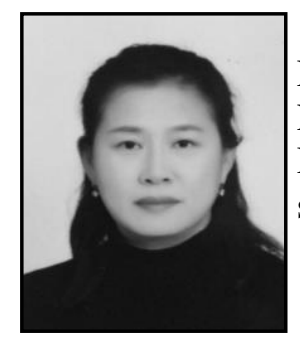

Hye-Jeong Song, she received the Ph.D. Degree from the Department of Computer Engineering in Hallym University. She is a Research Professor of the Department of Convergence Software, Hallym University. Her research interests focus on biomedical system and bioinformatics.

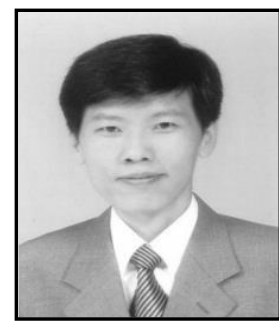

Chan-Young Park, he received the B.S. and M.S. from Seoul National University and the Ph.D. degree from Korea Advanced Institute of Science and Technology in 1995. From 1991 to 1999 he worked at Samsung Electronics. He is currently a Professor of the Department of Convergence Software, Hallym University. His research interests are in Bio-IT convergence, Intelligent Transportation System, and Sensor Networks.

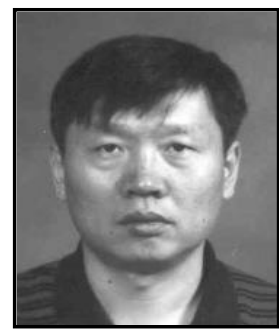

Jong-Dae Kim, he received the M.S. and the Ph.D. degree from Electrical Engineering from Korea Advanced Institute of Science and Technology, Seoul, Korea, in 1984 and 1990. He worked for Samsung Electronics from 1988 to 2000 as an electrical engineer. He is a Professor of the Department of Convergence Software, Hallym University. His research interests focus on biomedical system and bioinformatics. 


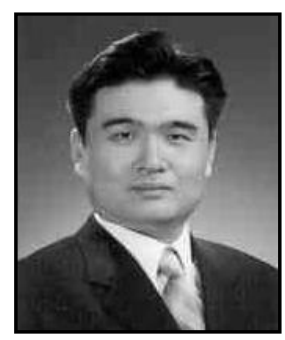

Yu-Seop Kim, He received the the Ph.D. degree from Computer Engineering from Seoul National University. $\mathrm{He}$ is currently a Professor in the Department of Convergence Softqare at Hallym University, South Korea. His research interests are in the areas of bioinformatics, computational intelligence and natural language processing. 